\title{
UNA VISIÓN PANORÁMICA DE LAS EXPERIENCIAS DE TELESALUD EN PERÚ
}

\author{
Ernesto Gozzer Infante1, $1,2, a$
}

\begin{abstract}
RESUMEN
El artículo presenta las principales experiencias de telesalud realizadas en el Perú entre los años 2002 al 2010. Analiza información de literatura publicada y documentos no publicados acerca de experiencias de telesalud públicas y privadas. A partir de esta información se consultó con 26 expertos acerca de las características de las experiencias de telesalud identificadas. Se generó una base de datos de 38 experiencias, que se describen y analizan en esta publicación. Se presenta reflexiones y recomendaciones para ampliar el uso de la telesalud en el Perú.
\end{abstract}

Palabras clave: Telesalud; Telemedicina; Informática médica (fuente: DeCS BIREME).

\section{AN OVERVIEW OF TELEHEALTH EXPERIENCES IN PERU}

\begin{abstract}
This article presents the main experiences of telehealth conducted in Peru between 2002 and 2010. It analyzes information from published literature and unpublished documents about experiences of public and private Telehealth. From this information, 26 experts were consulted about the characteristics of the identified Telehealth experiences. A database of 38 experiences, which are described and analyzed in this publication was produced. Reflections and recommendations are presented to expand the use of Telehealth in Peru.
\end{abstract}

Key words: Telehealth; Telemedicine; Medical informatics (source: MeSH NLM).

\section{INTRODUCCIÓN}

Se denomina telesalud al uso de tecnologías de avanzada en telecomunicaciones para intercambiar y brindar servicios de atención en salud, solucionando barreras geográficas, temporales, sociales y culturales. Mientras que el término telemedicina se circunscribe a servicios de atención médica directa, telesalud denota una definición más amplia ${ }^{(1)}$.

La Organización Mundial de la Salud (OMS) caracteriza a la telemedicina por proveer soporte clínico, conectar usuarios que no se encuentran en la misma ubicación física, e involucrar varios tipos de tecnologías de información y comunicación (TIC), para mejorar los resultados en salud (2).

La salud móvil, mhealth, es un componente de la telesalud que utiliza dispositivos como teléfonos móviles, dispositivos personales de monitoreo de funciones vitales, computadoras de bolsillo actualmente en desuso (PDA) y otros dispositivos inalámbricos. Hace uso de las funciones del celular (mensajes de texto, correos de voz), y funcionalidades más complejas, como el servicio general de paquetes vía radio (GPRS), telecomunicaciones móviles de tercera y cuarta generación ( $3 G$ y $4 G$ ), sistema de posicionamiento global (GPS) y Bluetooth (3). En el 2013, aunque variable, el acceso a estas tecnologías era bastante alto en el Perú, así en septiembre de ese año, el 22,5\% de las personas tenían acceso a Internet en los hogares, mientras que el $83,4 \%$ contaban con teléfono celular (4). Con el crecimiento del acceso a Internet por medio de los teléfonos celulares, el acceso real a Internet está creciendo de manera acelerada en los últimos años.

La telesalud permite a los servicios llegar donde las personas se encuentran, donde viven, trabajan y requieren atención. Esta herramienta, es útil para la recuperación de la salud, la detección precoz, la prevención de daños y riesgos y la promoción de estilos de vida saludables. El uso de estas tecnologías ha

\footnotetext{
Instituto Nacional de Salud. Lima, Perú.

Universidad Peruana Cayetano Heredia, Lima, Perú

Médico, MSc

Recibido: : 30-04-15 Aprobado: 13-05-15
}

Citar como: Gozzer Infante E. Una visión panorámica de las experiencias de telesalud en Perú. Rev Peru Med Exp Salud Publica. 2015;32(2):385-90. 
demostrado ser costo-efectivo, disminuyendo el costo de transporte ${ }^{(5,6)}$.

\section{EXPERIENCIAS DE TELESALUD EN PERÚ}

El presente artículo identifica las experiencias y proyectos de telesalud desarrollados en el Perú a partir del año 2002. Se realizó una exhaustiva búsqueda de literatura no publicada, así como de literatura publicada en bases de datos como Pubmed y Scielo, utilizando como palabras clave telesalud, telemedicina, telegestión, salud móvil; Telehealth, Telemedicine, Telemanagement, mHealth y Perú.

A partir de la información recogida, se diseñó un cuestionario para expertos con el fin de identificar experiencias de telesalud del sector público y privado. El instrumento indagaba acerca de las características de las experiencias seleccionadas, incluyendo: tipo de proyecto de telesalud, tecnología y equipos usados, fuente y tipo de financiamiento, ubicación geográfica del proyecto, área de salud objetivo de la intervención, y estado de actividad. Asimismo, se consultó a los expertos acerca del grado de conocimiento de normas nacionales y de adopción de la telesalud entre los prestadores de servicios de salud. Por último, se recogió sus recomendaciones respecto al rol del sector público y privado y las formas de solucionar las dificultades de implementación identificadas.

El cuestionario se aplicó en formato virtual a 26 expertos. La información recogida se consolidó en una base de datos de 38 proyectos que se presenta en la Tabla 1.

El tipo de proyectos de telesalud que se desarrolla en el Perú es muy variado; incluye desde iniciativas de educación a distancia de profesionales de la salud utilizando páginas web y transmisión de datos por Internet, hasta asesoramiento de emergencias neonatales en línea desde Lima a hospitales de regiones con exámenes de apoyo al diagnóstico por imágenes. Existen experiencias de uso de múltiples tecnologías de información y comunicación para el monitoreo de programas de salud, utilización de Internet para entrega de resultados de laboratorio, envío de imágenes de cultivos que son analizadas remotamente para generar el diagnóstico, identificación de epidemias, mejora de la adherencia de pacientes crónicos, incluyendo detector de caídas en pacientes que se recuperan en su domicilio. Algunas de las iniciativas han logrado sostenerse en el tiempo y ya cuentan con más de diez años de operación mientras que otras están recién iniciándose.
La mayor parte de los proyectos corresponden a telemedicina (58\%) y telegestión $(37 \%)$. En cambio el uso de TIC para información, educación y comunicación en salud en intervenciones preventivo-promocionales, representa solo el $21 \%$ del total de proyectos. La teleducación dirigida a mejorar capacidades de los profesionales y trabajadores de salud comprende solo un $12 \%$. La mayor parte de intervenciones de telemedicina usa sesiones interactivas en vivo (59\%), seguido por interacciones en diferido (32\%), y en menor proporción, el monitoreo remoto y monitoreo de pacientes mediante mensajes de texto.

El canal o medio de comunicación más frecuentemente utilizado es Internet (95\%) combinado, la mayor parte de las veces, con mensajes de texto SMS (32\%), mensajes interactivos de voz IVR (18\%), o voz (16\%) (Figura 1). La mención de radio hace pensar en la creatividad para interconectar aquellos lugares en los que no llega otro medio moderno. Por otro lado, el uso de Bluetooth sugiere el uso creciente de dispositivos para medir patrones fisiológicos o valores de algunas pruebas de laboratorio, como tensiómetros o glucómetros. La forma de comunicación fue, en la mayoría de los casos, el texto escrito (76\%), seguido en menor proporción, por audio (45\%), imagen (45\%), y video (24\%).

El equipo más utilizado para enviar y recibir información es la computadora (89\%), seguido por el smartphone (32\%), el celular básico (32\%) y las tabletas (29\%). Es interesante observar que también se reporta el uso de teléfonos fijos o públicos como medio de intercambio información (21\%). En algunos casos, se ha usado la radio ( $8 \%$ ), así como el Point of Sale (POS) que es un dispositivo para cargar consumo con tarjetas de crédito/ débito (3\%). En el mediano plazo, probablemente estos últimos sean reemplazados por los smartphone de gama media o baja.

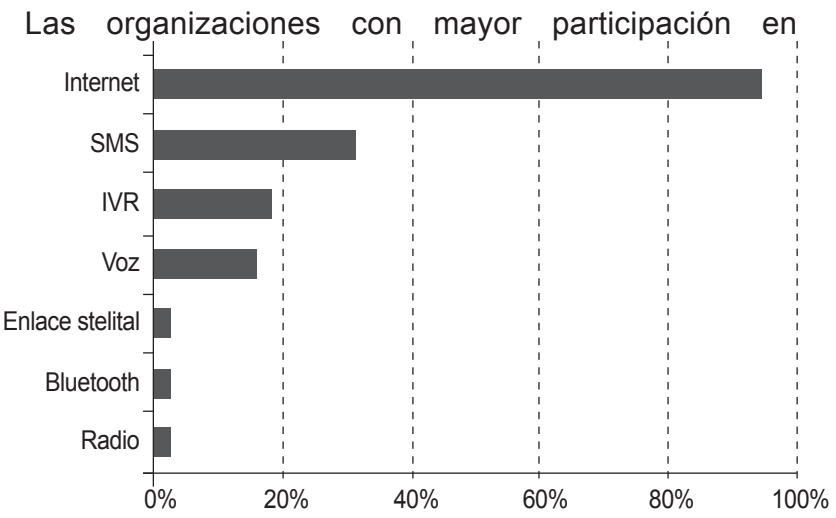

Figura 1. Canal de comunicación utilizado por los proyectos de telesalud del Perú 2002-2013

Fuente: Parsalud II 
Tabla 1. Proyectos de Telesalud en el Perú

\begin{tabular}{|c|c|c|c|c|c|c|c|c|c|c|c|c|c|c|}
\hline \multirow[b]{2}{*}{$\mathrm{N}^{\circ}$} & \multirow[b]{2}{*}{ Nombre } & \multicolumn{7}{|c|}{ Tipo de Tele salud } & \multicolumn{6}{|c|}{ Área Temática } \\
\hline & & $\begin{array}{l}\text { D } \\
\stackrel{0}{0} \\
\stackrel{0}{U} \\
\stackrel{5}{J} \\
\sqcup\end{array}$ & : & 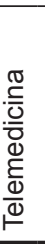 & 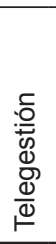 & $\begin{array}{l}\circlearrowleft \\
\stackrel{\varpi}{\Phi} \\
\frac{\Phi}{\oplus}\end{array}$ & 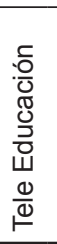 & 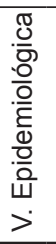 & 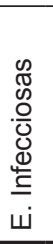 & 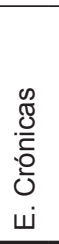 & 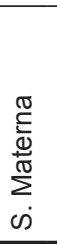 & 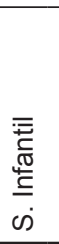 & 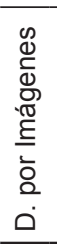 & $\stackrel{\text { o }}{\stackrel{2}{0}}$ \\
\hline 1 & $\begin{array}{l}\text { Mejora de la salud materno-infantil en la cuenta del rio Napo mediante el } \\
\text { uso apropiado de las TIC }\end{array}$ & Si & $\mathrm{R}$ & $\mathrm{X}$ & & & & $\mathrm{X}$ & $\mathrm{X}$ & & & & & \\
\hline 2 & Diagnóstico de enfermedades olvidades utilizando tele-microscopía de bajo coste & $\mathrm{Si}$ & $\mathrm{R}$ & $x$ & & & & $\mathrm{x}$ & $\mathrm{x}$ & & $\mathrm{x}$ & $\mathrm{x}$ & & \\
\hline 3 & $\begin{array}{l}\text { mejora de la salud materno infantil mediante un sistema de } \\
\text { teleestetoscopia de Perú }\end{array}$ & No & $\mathrm{R}$ & $\mathrm{x}$ & & & & & & & $\mathrm{x}$ & $\mathrm{x}$ & & \\
\hline 4 & $\begin{array}{l}\text { Control de malaria en zonas fronterizas de la Región Andina. Un enfoque } \\
\text { Comunitario }\end{array}$ & Si & $\mathrm{R}$ & & $\mathrm{x}$ & $x$ & & $\mathrm{x}$ & & & & & & \\
\hline 5 & Proyecto EHAS@LIS & No & $\mathrm{R}$ & & $x$ & $\mathrm{x}$ & & $\mathrm{x}$ & & & $\mathrm{x}$ & $\mathrm{x}$ & & \\
\hline 6 & AUNA HIS & & $\mathrm{R}$ & $x$ & & & & & $\mathrm{x}$ & $\mathrm{x}$ & $\mathrm{x}$ & $\mathrm{x}$ & $x$ & \\
\hline 7 & Programa EHAS & & $\mathrm{R}$ & $x$ & & & & $x$ & $\mathrm{x}$ & & $\mathrm{x}$ & $\mathrm{x}$ & $x$ & \\
\hline 8 & Proyecto de telerradiología Centro Nacional de Lectura de imágenes CENALI & & $\mathrm{R}$ & $x$ & & & & & & & & & $x$ & \\
\hline 9 & Alerta MINSA & & $\mathrm{R}$ & & $\mathrm{x}$ & & & $x$ & & & & & & \\
\hline 10 & Telemedicina en Candarave, Tacna & & $\mathrm{R}$ & $x$ & & & & & & & & & & $x$ \\
\hline 11 & NACER & & $\mathrm{R}$ & & $x$ & & & $x$ & $\mathrm{x}$ & & $\mathrm{x}$ & $x$ & & $x$ \\
\hline 12 & CARENET & & $\mathrm{R}$ & $x$ & & $x$ & & & & $x$ & & & & \\
\hline 13 & Cuida tu salud móvil & & $\mathrm{R}$ & & & $x$ & & & & $x$ & & & & \\
\hline 14 & Tele ecografía robótica & & $\mathrm{R}$ & $x$ & & & & & & & $\mathrm{x}$ & & & $x$ \\
\hline 15 & WawaRed & & $\mathrm{R}$ & $x$ & & & & & & & $x$ & $x$ & & \\
\hline 16 & Sistema electrónico open-source de vigilancia epidemiológica VIGILA & & $\mathrm{N}$ & & & $\mathrm{X}$ & & $x$ & $\mathrm{x}$ & & $\mathrm{x}$ & & & $x$ \\
\hline 17 & NETLAB Citología & & $\mathrm{R}$ & & $x$ & & & & & $\mathrm{x}$ & $\mathrm{x}$ & & & \\
\hline 18 & Sistema de vacunación FAP & & $\mathrm{N}$ & & $\mathrm{X}$ & & & & & & & $x$ & & \\
\hline 19 & NETLAB & & $\mathrm{N}$ & & $\mathrm{X}$ & & & & $\mathrm{x}$ & $x$ & & & & \\
\hline 20 & SINTB: Sistema de registro médico electrónico en el nivel local & & $\mathrm{R}$ & & $X$ & & & & $x$ & & & & & \\
\hline 21 & ALERTA DISAMAR & No & $\mathrm{N}$ & & $\mathrm{X}$ & & & $\mathrm{X}$ & & & & & & \\
\hline 22 & C@renet & No & $\mathrm{R}$ & $\mathrm{X}$ & & & & & & $\mathrm{X}$ & & & & \\
\hline 23 & Mtb & $\mathrm{Si}$ & $\mathrm{R}$ & $X$ & & & & & $\mathrm{X}$ & & & & & \\
\hline 24 & $\begin{array}{l}\text { Inter Support Action (International Network of Teleconsultation Excellence } \\
\text { Referral), Isa Platform Perú National Network }\end{array}$ & $\mathrm{Si}$ & $\mathrm{R}$ & $\mathrm{X}$ & & & & & $\mathrm{X}$ & $\mathrm{X}$ & & $\mathrm{X}$ & $\mathrm{X}$ & $\mathrm{X}$ \\
\hline 25 & Programa de telemedicina en EsSalud "Tele EsSalud" & $\mathrm{Si}$ & $\mathrm{R}$ & $\mathrm{X}$ & $\mathrm{X}$ & & $\mathrm{X}$ & & $\mathrm{X}$ & $\mathrm{x}$ & $\mathrm{X}$ & & $\mathrm{X}$ & \\
\hline 26 & $\begin{array}{l}\text { Use of Mobile Technology to PreventProgression of Pre-hypertension in } \\
\text { Latin American Urban Settings }\end{array}$ & No & $\mathrm{R}$ & & & $\mathrm{X}$ & & & & & & & & $\mathrm{X}$ \\
\hline 27 & Consultas oftalmológicas en línea, plan piloto sierra central & $\mathrm{Si}$ & $\mathrm{R}$ & $\mathrm{X}$ & & & & & & & & & & $\mathrm{X}$ \\
\hline 28 & E-ChASQUI & $\mathrm{Si}$ & $\mathrm{R}$ & & $X$ & & & & $\mathrm{X}$ & & & & & \\
\hline 29 & CellPOS & No & $\mathrm{R}$ & $X$ & & & & & $\mathrm{X}$ & & & & & \\
\hline 30 & CellPREVEN & No & $\mathrm{R}$ & & $\mathrm{X}$ & & & & $\mathrm{X}$ & & & & & \\
\hline 31 & Proyecto SOMOS & No & $\mathrm{R}$ & & & $\mathrm{X}$ & & & $\mathrm{X}$ & & & & & \\
\hline 32 & $\begin{array}{l}\text { Proyecto tu Nexo: Randomized controlled trial to evaluate the effect of a } \\
\text { combined web-based intervention to increase HIV testing in men who have } \\
\text { sex with men in Lima-Perú }\end{array}$ & $\mathrm{Si}$ & $\mathrm{R}$ & & & $\mathrm{X}$ & & & $\mathrm{X}$ & & & & & \\
\hline & Mednet Perú & No & $\mathrm{R}$ & $\mathrm{X}$ & & & & & & & $\mathrm{X}$ & $\mathrm{X}$ & $\mathrm{X}$ & \\
\hline & e-Prevención en América Latina y el Caribe & $\mathrm{Si}$ & N/D & $\mathrm{X}$ & & & & & $\mathrm{X}$ & & & & & \\
\hline 35 & CENATE (Centro Nacional de Telemedicina) & $\mathrm{Si}$ & $\mathrm{R}$ & $\mathrm{X}$ & & & & & & & & & $\mathrm{X}$ & $\mathrm{X}$ \\
\hline & $\begin{array}{l}\text { Telediagnóstico de tuberculosis y determinación de TB-MDR basado en el } \\
\text { método MODS }\end{array}$ & $\mathrm{Si}$ & $\mathrm{R}$ & $\mathrm{X}$ & & & & & $\mathrm{X}$ & & & & & \\
\hline & $\begin{array}{l}\text { Fortalecimiento de la adherencia al tratamiento DOTS mediante el uso } \\
\text { mensajes de texto recordatorios }\end{array}$ & $\mathrm{Si}$ & $\mathrm{R}$ & $\mathrm{X}$ & & & & & & $\mathrm{X}$ & & & & \\
\hline 38 & Red de Asitencia Técnica Virtual en Salud Materna y Neonatal INMP & $\mathrm{Si}$ & $\mathrm{N}$ & $\mathrm{X}$ & & & $\mathrm{X}$ & & & & $\mathrm{X}$ & $\mathrm{X}$ & & \\
\hline
\end{tabular}

R: regional; N: nacional; S.: salud; V.: vigilancia; E.: enfermedad; Tic: tecnologías, información y comunicación; IEC: información, educación, comunicación; TB-MDR: tuberculosis multidrogoresistente; MODS: observaciónmicrsocópica y suceptibilidad a chagas. 


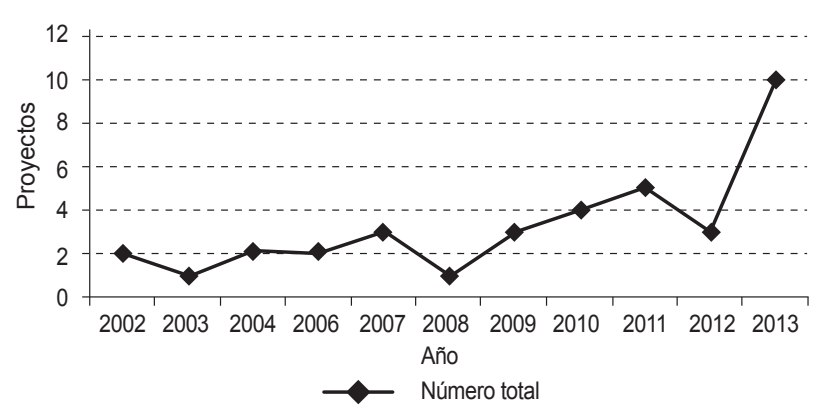

Figura 2. Evolución del número de proyectos de telesalud en el Perú 2002-2013

Fuente: Parsalud II

el desarrollo de este campo como financiadoras, desarrolladoras, implementadoras o conductoras pertenecen a varios sectores: la academia (UPCH), el sector privado (Voxiva), la cooperación internacional como Naval Medical Research Unit (NAMRU), Enlace Hispano Americano en Salud (EHAS), Convenio Hipólito Unánue (CONHU), el sector público como el INS, la Sanidad de Fuerzas Armadas, EsSalud y el Instituto Nacional Materno Perinatal. De manera creciente se observa la participación de otras organizaciones del sector público (PARSALUD II- MINSA, DIRESA) y también del sector privado (Grupo AUNA). Pero también hay esfuerzos individuales (Proyecto Tele Oftalmología).

Los proyectos de telesalud estudiados están dirigidos a atender la salud materno infantil $(71 \%)$, enfermedades infecciosas (45\%), vigilancia epidemiológica $(24 \%)$ y enfermedades crónicas $(21 \%)$ tal como se observa en la Figura 2. Estos proyectos también se ocupan del diagnóstico por imágenes (18\%). No se reporta proyectos en el área de salud mental como tema de Telesalud, a pesar de la alta carga de enfermedad que genera en nuestro país y la existencia de este tipo de intervenciones en el contexto internacional.

Los proyectos generalmente se desarrollan en Lima, seguidos por Loreto, La Libertad, Junín, y otras nueve regiones. El financiamiento de los proyectos o experiencias proviene principalmente de recursos propios $(32 \%)$ o cooperación internacional $(29 \%)$. Un número importante de proyectos han sido financiados por medio de concursos internacionales $(21 \%)$ y nacionales $(16 \%)$.

Respecto al uso de certificaciones, estándares y protocolos internacionales, se encontró que la mayoría de proyectos de telemedicina reporta utilizar los estándares de datos del Ministerio de Salud (21\%). En cambio, se usa de manera incipiente otros estándares como Digital Imaging and Communication in Medicine, DICOM (13\%), Picture Archiving and Communications Systems PACS (11\%) y Radiology Information System RIS (8\%). Un porcentaje importante no usa o no conoce $(29 \%)$ estos estándares. Al parecer solamente AUNA y EsSalud han avanzado en el uso sistemático de las certificaciones y estándares nacionales e internacionales.

Si bien los dos primeros proyectos de telesalud en el Perú empiezan a funcionar en el 2002, su número aumenta en el 2011 y el 2013, como se observa en el Figura 2. De todos los proyectos registrados, la mayoría siguen funcionando $(66 \%)$.

A partir de las variadas experiencias de telesalud presentadas, podemos afirmar que en el Perú existe habilidad en el uso de tecnologías, interés y creatividad. Por ejemplo, el proyecto MedNet Perú, implementado en Junín con financiamiento de la Unión Europea, reporta el uso de Smartphones, por parte de médicos, para enviar imágenes al consultar por una segunda opinión. En muchos países se ha demostrado la utilidad de la telesalud para compensar la ausencia de recursos humanos especializados en este campo y contribuir a un acceso equitativo a la salud ${ }^{(7,8)}$. Muchas de las regiones de nuestro país podrían beneficiarse de la consulta por Telepresencia o segunda opinión a especialistas. Se puede aprender de la experiencia de las consultas virtuales que realiza el Instituto Nacional Materno Perinatal INMP en neonatología, especialmente la que se está realizando con el apoyo del PARSALUD II en Ucayali, Amazonas y Huánuco regiones en las que no existe neonatólogo.

De igual manera, El Instituto Nacional de Salud desarrolló NETLAB, que es una herramienta de gestión de los datos de laboratorio para clínicos y usuarios. Se inició con personas que viven con VIH/SIDA y después se amplió a cerca de cien enfermedades ${ }^{(9)}$.

Las herramientas de telesalud pueden adaptarse a los distintos contextos culturales y sanitarios como evidencia un estudio dirigido a mejorar la adherencia al tratamiento con el uso de mensajes de texto que destaca la importancia de personalizar los mensajes y adecuar las intervenciones a los grupos de población y problemas específicos ${ }^{(10)}$.

La integración de las tecnologías de información y comunicación con el sistema de servicios de salud, es un proceso que está redefiniendo el futuro de la atención de salud en el mundo (11). En el Perú se debe asumir este reto aprendiendo las lecciones de experiencias realizadas en el país y las de los países que han logrado extender su aplicación al ámbito nacional. 
El apoyo político de los responsables institucionales es un elemento fundamental para el desarrollo de la Telesalud. Los procesos de implementación de Telesalud son usualmente largos porque comprenden el diseño de respuestas que utilizan nuevas formas de aplicar tecnología, la conformación de verdaderos equipos multidisciplinarios, un conjunto de arreglos institucionales que incluyen mecanismos legales y nuevas formas de financiamiento. Comenzar a evidenciar los beneficios toma un tiempo que los directivos no siempre están dispuestos a aceptar. Este proceso debe tener en cuenta que la mayoría de las intervenciones de Telesalud tienen un período de maduración relativamente largo. Por tanto, es importante promover la estrategia de desarrollo de innovaciones locales o experiencias iniciales que luego de ajustarse, permitirán su expansión regional o nacional.

Es necesario que la autoridad sanitaria asuma liderazgo tanto intra como intersectorial definiendo mecanismos que permitan ordenar el crecimiento de esta herramienta para el desarrollo de intervenciones en salud. De esta manera, se potencia la eficiencia de las inversiones con un adecuado balance costo-efectividad. Resulta urgente revisar las normas nacionales de Telesalud con la experiencia nacional acumulada y la capacidad tecnológica disponible en el presente. De manera especial se debe renovar, de manera periódica, las certificaciones y estándares obligatorios que deben tener las intervenciones que utilicen tecnologías de información y comunicación para la salud. Otro documento que requiere ser actualizado, es el Plan Nacional de Telesalud, que debe incluir la definición de prioridades de intervención que van a promover el desarrollo de la Telesalud.

Desde el punto de vista de la infraestructura tecnológica, aún se requiere el aumento del ancho de banda y de la cobertura de acceso, en particular a poblaciones de las regiones amazónica y andina. Sin embargo, la situación actual hace factible el desarrollo de intervenciones de cobertura nacional, como es el caso de Alerta DISAMAR ${ }^{(12,13)}$.

Por último, se observa que pocos actores conocen lo que otros están haciendo. En este sentido, se recomienda la promoción de redes de intercambio de información y la utilización de diferentes mecanismos como foros, reuniones de intercambio de experiencias, simposios y publicaciones periódicas. Por ejemplo, resulta interesante encontrar que la Dirección de Salud de la Marina de Guerra del Perú utilice un moderno sistema de vigilancia epidemiológica electrónica -Alerta DISAMAR que integra el uso de Internet, teléfonos fijos y celulares. Este sistema se ha expandido a otras sanidades militares en el Perú y varios países de África y Latinoamérica ${ }^{(14)}$. Sin embargo, la experiencia no ha sido replicada en Perú en otros ámbitos sanitarios.

Habiéndose iniciado un banco de proyectos y experiencias de Telesalud, es recomendable establecer un banco de datos que registre los datos de proyectos para contar con información actualizada. Para ello, se puede utilizar la misma o una versión revisada de la estructura utilizada en este estudio. El ingreso de datos y los resultados deberían ser mostrados el website del MINSA o del PARSALUD II. Se debe promover activamente que se registren ahí las experiencias que se desarrollen en este campo. Este registro de datos puede permitir no solo el conocimiento de la evolución de la Telesalud sino que puede ser un medio para el intercambio de experiencias y la colaboración entre diferentes actores.

\section{CONCLUSIONES}

Enconclusión, esta revisión poneen evidenciala necesidad de un trabajo colaborativo, de un esfuerzo institucional y de fondos para pasar de intervenciones innovadoras en el nivel local a intervenciones a nivel nacional con un enfoque sistémico que potencie lo ya desarrollado y promueva nuevos usos de la Telesalud para dar un mayor impacto a las políticas y estrategias en salud. Esperamos que la presentación de esta visión panorámica del campo contribuya al desarrollo de espacios y mecanismos para que los equipos multidisciplinarios que trabajan estos temas, se encuentren y compartan las lecciones generando una colaboración. Es decir, enseñando unos a otros lo aprendido y estimulando el desarrollo de ideas innovadoras que hagan uso del intercambio de texto, voz, imagen a distancia para resolver problemas de salud.

Agradecimientos: el presente artículo contó con el valioso apoyo del equipo del PARSALUD II, en particular de Elsa Cecilia Díaz Arroyo, Nelson Larrea Claros, María Colán Gómez, Mónica Romero Arzapalo, Rosa Inés Béjar Cáceres y Walter Vigo Valdez, quienes asistieron técnica y metodológicamente el desarrollo de la consultoría, cuyos contenidos forman parte del presente artículo.

Fuentes de financiamiento: este artículo utiliza parte del informe final de la consultoría "Sistematización de las experiencias piloto de Telesalud/Telemedicina en el país", elaborado por el autor con el financiamiento del Programa de Apoyo a la Reforma del Sector Salud -PARSALUD II. Su contenido es únicamente responsabilidad del autor y no representa necesariamente el punto de vista del PARSALUD II.

Conflictos de interés: el autor declara no tener conflictos de interés. 


\section{REFERENCIAS BIBLIOGRÁFICAS}

1. National Academy of Sciences. The Role of Telehealth in an Evolving Health Care Environment - Workshop Summary [Internet]. Washington, DC: The National Academies. 2015 [citado el 23 de setiembre del 2014]. Disponible en: http://www.iom. edu/en/Reports/2012/The-Role-ofTelehealth-in-an-Evolving-HealthCare-Environment

2. World Health Organization. Telemedicine: opportunities and developments in Member States: report on the second global survey on eHealth. Vol 2 [Internet]. Ginebra: WHO; 2010 [citado el 25 de setiembre del 2013]. Disponible en: http://www. who.int/goe/publications/ehealth series_vol2/en/

3. World Health Organization. mHealth: new horizons for health through mobile technologies: second global survey on eHealth. Vol 3 [Internet]. WHO: Geneva; 2011 [citado el 25 de setiembre del 2014]. Disponible en: http://www. who.int/goe/publications/ehealth_ series_vol3/en/index.html

4. Instituto Nacional de Estadística e Informática. Las Tecnologías de Información y Comunicación en los Hogares [Internet]. Lima: INEI; 2013 [citado el 25 de setiembre del 2014]. Disponible en: http://www. inei.gob.pe/media/MenuRecursivo/ boletines/17217.pdf
5. Bynum $\mathrm{AB}$, Irwin $\mathrm{CA}$, Cranford $\mathrm{CO}$, Denny GS. The impact of telemedicine on patients' cost savings: some preliminary findings. Telemed J E Health. 2003 Winter;9(4):361-7.

6. Oliveira TC, Barlow J, Gonçalves L, Bayer S. Teleconsultations reduce greenhouse gas emissions. J Health Serv Res Policy. 2013 Oct;18(4):20914. doi: 10.1177/1355819613492717.

7. Wamala DS, Augustine K. A metaanalysis of telemedicine success in Africa. J Pathol Inform. 2013 May 30;4:6. doi: $10.4103 / 2153-3539.112686$.

8. Bagayoko CO, Anne A, Fieschi M, Geissbuhler A. Can ICTs contribute to the efficiency and provide equitable access to the health care system in Sub-Saharan Africa? The Mali experience. Yearb Med Inform. 2011;6(1):33-8.

9. García PJ, Vargas JH, Caballero NP, Calle VJ, Bayer AM. An e-health driven laboratory information system to support HIV treatment in Peru: E-quity for laboratory personnel, health providers and people living with HIV. BMC Med Inform Decis. 2009;9:50.

10. Curioso WH, Alex Quistberg D, Cabello R, Gozzer E, Garcia PJ, Holmes KK, et al. "It's time for your life": How should we remind patients to take medicines using short text messages?. AMIA Annu Symp Proc. 2009;2009:129-33.

11. Eron L. Telemedicine: the future of outpatient therapy?. Clin Infect Dis. 2010 Sep 15;51 Suppl 2:S224-30. doi: $10.1086 / 653524$.

12. Soto G, Araujo-Castillo RV, Neyra J, Fernandez M, Leturia C, Mundaca CC, et al. Challenges in the implementation of an electronic surveillance system in a resource-limited setting: Alerta, in Peru. BMC Proc. 2008;2 (Suppl 3):S4.

13. Huaman MA, Araujo-Castillo RV, Soto G, Neyra JM, Quispe JA, Fernandez $\mathrm{MF}$, et al. Impact of two interventions on timeliness and data quality of an electronic disease surveillance system in a resource limited setting (Peru): a prospective evaluation. BMC Med Inform Decis Mak. 2009 Mar 10;9:16. doi: 10.1186/1472-6947-9-16.

14. Gozzer E. How Peru and Latin America are using mHealth. Exchange on HIV and AIDS Sexuality and Gender. 2012 Jul;2:14-5.

Correspondencia: Ernesto Gozzer

Dirección:Cápac Yupanqui 1400, Lima 11, Perú

Correo electrónico: ernesto.gozzer@gmail.com

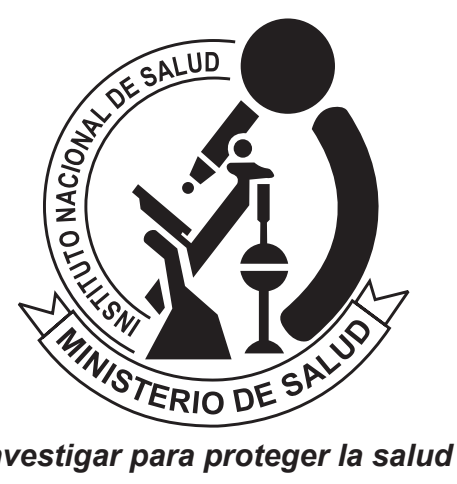

REVISTA PERUANA DE MEDICINA EXPERIMENTAL Y SALUD PÚBLICA CUMPLIENDO SUS METAS $Y$ PROYECTÁNDOSE AL FUTURO

Visite los contenidos de la revista en: www.ins.gob.pe/rpmesp 